\title{
Microdiseño de unidades didácticas aplicadas a la formación del especialista en Estomatología General Integral
}

\author{
Microdesign of teaching units applied to the training of specialists in \\ Comprehensive General Stomatology
}

\author{
Ana Caridad López Vantour ${ }^{1 a}$, María Elena Álvarez López ${ }^{2 b}$, Nadina Travieso Ramos ${ }^{2 b}$
}

\section{RESUMEN}

En la presente investigación se elaboró el microdiseño curricular de las unidades didácticas correspondientes al Módulo de atención al menor de 19 años I, correspondiente al plan de estudios de la Especialidad en Estomatología General Integral, el universo estuvo constituido por 59 residentes que cursaban el primer año durante el curso escolar 2018-2019, los resultados fueron analizados a través de la frecuencia absoluta y el porcentaje, para comprobar la factibilidad de la propuesta se aplico el estadígrafo Mac Nemar, comprobándose los cambios de antes y después de aplicado el instrumento ( prueba de desempeño), así como una encuesta de satisfacción a los residentes, los resultados obtenidos hacen de esta propuesta una alternativa para el perfeccionamiento del proceso enseñanza aprendizaje en la formación del posgrado.

Palabras claves: Educacion de Posgrado en odontologia; Estomatología; didáctica. (Fuente: DeCS BIREME)

\section{ABSTRACT}

In the present investigation the curricular microdiseño was elaborated from the didactic units corresponding to the Module of attention to the smaller than 19 years I, corresponding the plan of study of the specialty of General Integral Estomatología, the universe was constituted by 59 residents that studied the first year during the school course 2018-2019, the results they were analyzed through the absolute frequency and the percentage, to check the feasibility of the proposal you applies the statistician Mac Nemar, being proven the changes of before and after having applied the instrument (it proves of acting), as well as a survey of satisfaction to the residents, the obtained results make of this proposal an alternative for the improvement of the process teaching learning in the formation of the posgrado.

Key words: Eduacation, Dental, Graduate ; Stomatology; Didactic. (Source: MeSH NLM)

Recibido: 02 de febrero de 2020

Aprobado: 6 de julio de 2020

Publicado: 7 de octubre de 2020

${ }^{1}$ Facultad de Estomatología, Universidad de Ciencias Médicas.

Santiago de Cuba, Cuba.

${ }^{2}$ Hospital Militar Dr. Joaquín Castillo Duany Santiago de Cuba, Cuba.

${ }^{\text {a }}$ Docente

${ }^{\mathrm{b}}$ Odontóloga

Correspondencia:

Dra. Ana Caridad López Vantour

Correo electrónico: a.lopez @infomed.sld.cu
Este es un artículo Open Access distribuido bajo la licencia Creative Commons Atribución-NoComercialCompartirlgual 4.0

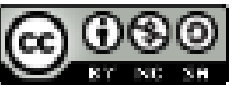

Citar como: López Vantour AC, Álvarez López ME, Travieso Ramos N. Microdiseño de Unidades didácticas aplicadas a la formación del especialista en Estomatologia Integral. KIRU. 2020; 17(4): 194-200. https://doi.org/10.24265/kiru.2020.v17n4.3 


\section{INTRODUCCIÓN}

La educación de posgrado o cuarto nivel de enseñanza, es considerado como el nivel más alto del Sistema Nacional de Educación en Cuba; se basa en el desarrollo profesional a travês de cursos como los diplomados y a la formación académica como las especialidades, maestrías y doctorados) ${ }^{(1)}$.

A nivel internacional, los sistemas educativos en el posgrado pueden variar, sin embargo, la estructura curricular se caracteriza por ser interdisciplinaria, multidisciplinaria, flexible y estar centrada en el alumno. Mientras que en las estrategias de enseñanza - aprendizaje, se resalta la inovación, la creacion de conocimientos, el analisis critico, la autonomia intelectual, el rigor del trabajo académico, el analisis y procesamiento de informacióny la solución de problemas. ${ }^{(2)}$.

El Reglamento de la Educación de Posgrado de la República de $\mathrm{Cuba}^{3}$, al referirse a la especialización de manera general, plantea que esta proporciona la actualización, profundización, perfeccionamiento o ampliación de las competencias laborales para el desempeño profesional que requiere un puesto de trabajo. Por otro lado, al referirse a la especialización en salud, plantea como objetivo que cada profesional actulice sus conocimientos según las funciones de trabajo, a fin que este preparado para solucionar nuevos problemas de salud, el apoyo a las investigaciones según los cambios en la situación de salud de cada territorio y los adelantos científicos tecnológicos actuales ${ }^{(3)}$.

Desde el siglo XIX existe en el mundo la especialización en la práctica médica cuya característica fundamental es de aprender haciendo, concebida como un sistema donde el aprendizaje se construye a través de la práctica en un servicio de salud bajo supervisión permanente de un tutor o docente con la asignación de responsabilidades crecientes, al mismo tiempo, que se adquieren los hábitos del trabajo de la profesión en cuestión (4). En esta misma línea de pensamiento la formación de un especialista en Cuba, como una forma de la educación de posgrado en salud, tiene como objetivo básico formar profesionales de la salud con alto nivel de desempeño, solidez en los principios éticos morales de la sociedad cubana y elevada calificación científico-técnica, capaces de responder a las características de la universidad innovadora cubana: científica, tecnológica y humanista, con un fuerte compromiso social que garantiza la calidad de la atención, el cambio del estado de salud y la satisfacción de la población. ${ }^{(3)}$.

En tanto la referirse a la Estomatología, las especialidades comenzaron en el año 1962, las del segundo nivel de atención, o también llamadas especialidades clínicas, (Cirugía Máxilo- Facial, Ortodoncia, Periodoncia y Prótesis dental), hasta que con la implementación del Programa del médico y enfermera de la familia surge la especialidad de Estomatología General Integral (EGI), como nuevo modelo de atención estomatològica dentro de la Atención Primaria de Salud, lo cual representa un pilar significativo dentro del Sistema Nacional de Salud, para dar respuestas a las demandas sociales tanto en Cuba como a nivel internacional ${ }^{(5)}$. La especialidad de EGI se desarrolla con un enfoque biosico social en la solución de los problemas de salud bucal del individuo, la familia y la comunidad, donde prevalecen las acciones de promoción de salud y prevención de enfermedades integrando lo biológico y lo social; lo preventivo y lo curativo, estrechamente vinculado con el entorno físico, biológico y social en el cual vive el hombre.

Desde una perspectiva académica, para la formación de este especialista en el proceso enseñanza aprendizaje (PEA), se conciben diferentes formas organizativas de enseñanza como conferencias, seminarios, clases taller, discusión de problemas de salud, revisiones bibliográficas, y la práctica en servicio, o Educación en el Trabajo todas a punto de partida del proceso salud enfermedad, esta última favorece una atención estomatológica integral que incluye acciones de promoción, prevención, curación y rehabilitación, conjuntamente con otras funciones, como gerencial, e investigativa que contribuye a egresar un profesional con un nivel de competencia colaborando con la calidad de los servicios que se prestan ${ }^{(6)}$.

Todo lo anteriormente planteado permite reconocer que la formación del especialista en Cuba se desarrolla y concreta "en" y "a través" de las acciones de la atención en salud mediante la Educación en el Trabajo en las unidades y servicios en que se desempeñan, donde la atención primaria de 
salud constituye la estrategia central de su ejecución. Los procesos de formación del especialista se diseñan a fin de garantizar el desarrollo de cuatro funciones esenciales para el trabajo profesional en el Sistema Nacional de Salud (SNS): atencionales, educacionales, investigativas y de gestión en salud. Lo cual conlleva a reconocer el papel de la Didáctica especializada de las Ciencias de la Educación Médica en la formación del especialista en este caso en $\mathrm{EGI}$, ya que permite establecer un sistema de relaciones interactivas, complejas y sistémicas entre el profesor, el residente y el grupo; lo que favorece el paradigma educacional actual de aprender a aprender, y de aprender haciendo bajo la conducción del que enseña, que debe dirigir los espacios necesarios para que los residentes desarrollen su actitud competente.

Desde ese punto de vista, los docentes no deben limitarse a enseñar contenidos conceptuales y procedimentales, sino que es necesario integrar actividades que permitan una interacción ciencia-tecnología-sociedad, que valoren aspectos que permitan la solución de problemas propios de la profesión, sin embargo, se mantienen insuficiencias en la concepción didáctica que se emplea en el PEA de los módulos en la especialidad de EGI, lo cual se declara como problema de la investigación ${ }^{(7)}$.

En la solución de esta problemática a nivel de microdiseño curricular se puede utilizar como alternativa, el diseño de unidades didácticas. Entendiéndose como unidad didáctica como una forma de planificar el proceso de enseñanza-aprendizaje alrededor de un elemento de contenido que se convierte en eje integrador del proceso y le aporta consistencia y significatividad ${ }^{(8)}$.

Sánchez y Valcárcel ${ }^{8}$ diseñaron el modelo de planificación de unidades didácticas donde sus componentes son los siguientes: selección del objetivo, análisis del contenido (selección y estructuración de conocimientos, habilidades y valores), selección de estrategias didácticas (planteamientos metodológicos, formas de organización de las actividades de enseñanza y medios de aprendizaje), así como selección de estrategias de evaluación. Sin embargo, para Sanmartí diseñar una unidad didáctica para llevarla a la práctica, es decidir qué se va a enseñar y como es la actividad mas importante que llevamos a cabo los enseñantes, ya que de ella concretamos y ponemos en práctica nuestras ideas e intenciones educativas ${ }^{(9)}$. Por otro lado, Bautista Perdomo ${ }^{9}$ considera, la Unidad Didáctica como una actividad compleja, que demanda la elección y estructura de los contenidos de enseñanza, el análisis de actividades de enseñanza, la evaluación del aprendizaje de los estudiantes y la revisión histórica-epistemológica de los conceptos.

El presente trabajo está dirigido al microdiseño curricular de las unidades didácticas correspondientes al Módulo Atención Estomatológica al menor de 19 años I, y evaluación de la factibilidad de su aplicación a través de una prueba de desempeño realizada a los residentes que cursaban el primer año de la especialidad durante el curso escolar 2018-2019, en la Facultad de Estomatología de Santiago de Cuba.

\section{MATERIALES Y MÉTODO}

Se realizó un estudio cuasi experimental, donde se aplicó el estadígrafo Mac Nemar, para la evaluación de los cambios de antes y después de aplicada la prueba de desempeño; así como la factibilidad de la aplicación de las unidades didácticas El universo estuvo constituido por 59 residentes que cursaban el primer año de la especialidad de EGI, durante el curso 2018-2019.

Para la realización del presente estudio se emplearon métodos teóricos (análisis y síntesis, ascensión de lo abstracto a lo concreto, así como el método sistémico estructural) y empírico (revisión documental y encuesta de satisfacción).

La revisión documental se realizó al plan de estudios de la citada especialidad lo cual permitió seleccionar el sistema de conocimientos, habilidades y valores, las propuestas metodológicas, medios de enseñanza a utilizar y el sistema de evaluación.

En la planificación de las unidades didácticas, el hilo conductor estuvo dado por el carácter sistémico, la coherencia y el carácter lógico del diseño teórico-metodológico de la investigación educativa en salud, paralelamente cada una de las unidades didácticas tenía un objetivo en correspondencia con el sistema de conocimiento. Como procedimientos metodológicos se utilizaron situaciones de enseñanza aprendizaje propios de la profesión, para el desarrollo del pensamiento 
reflexivo, la independencia cognoscitiva y la creatividad con un carácter interdisciplinario, así como el uso de las TICs, como medio de enseñanza y soporte material del proceso de enseñanza - aprendizaje.

Se concibió la evaluación formativa de manera cualitativa para cada una de las unidades didácticas, y una evaluación final. La factibilidad de su aplicación se evaluó a través de los resultados de la prueba de desempeño, así como los resultados de la encuesta de satisfacción realizada. Para el resumen de los datos se utilizó la frecuencia absoluta y el porcentaje, la prueba de MacNemar con un nivel de significación estadística de 0,05 . Los resultados se expresan en tabla1 figura1.

\section{RESULTADOS}

Inicialmente antes de utilizar las unidades didácticas se les aplicó el instrumento a 59 residentes, de cuales solo resultaron aprobados 3 residentes, y 56 desaprobados, después de la utilización de las unidades didácticas y aplicar nuevamente el instrumento se obtiene que, de 56 desaprobados, 51 cambian su evaluación para aprobados y solo 5 desaprueban, como resultado final se obtiene un 96,42 \% de aprobados, resultado significativo.

Tabla 1. Resultados de antes y después de aplicadas las unidades didácticas

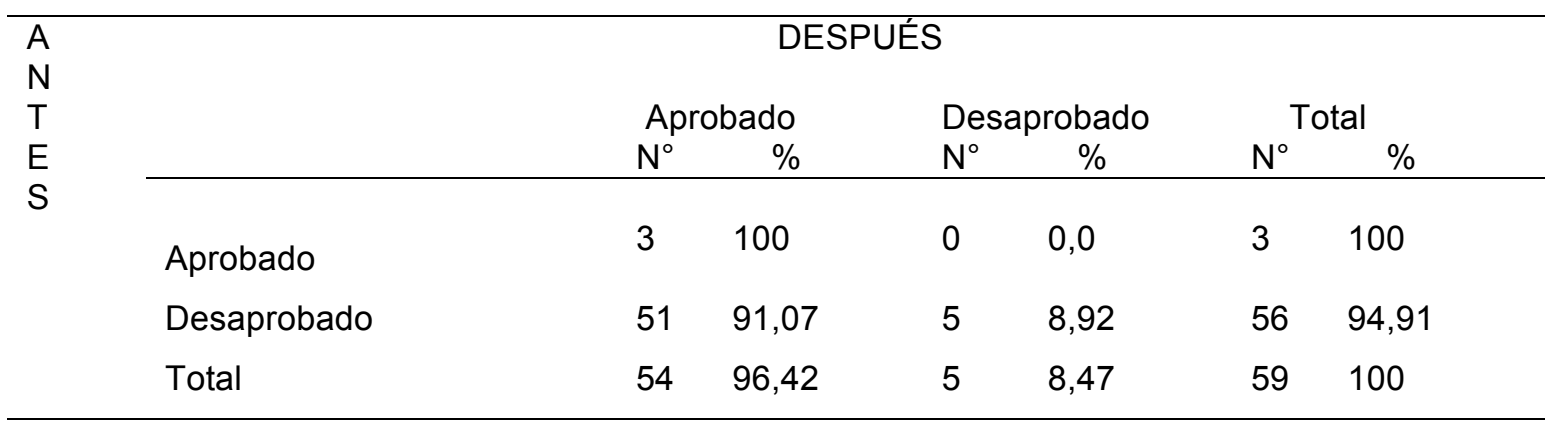

Nivel de significancia $(p>0,05)$

Con relación a la satisfacción, se aplicó una encuesta de siete preguntas relacionadas con los aspectos que conformaban las unidades didácticas, se utilizaron las categorías de muy satisfecho, satisfecho, poco satisfecho, no satisfecho y no puedo responder.

Se obtiene como resultado que $84,74 \%$ estaba muy satisfecho con relación a la utilización de las unidades didácticas, y el $15,25 \%$ satisfecho, con relación a la situaciones de enseñanza - aprendizajes propios de la profesión, con un carácter interdisciplinario, el $96,61 \%$ estaba muy satisfecho y solo el $5,08 \%$ eran satisfecho, mientras que el $100 \%$ se encontró muy satisfecho con relación a las habilidades, unido al uso de las TIC como medio de enseñanza, mientras que el $100 \%$ estuvo satisfecho en relación con los valores desarrollados y el sistema de evaluación, empleado; finalmente el 96,61 \% estuvo muy satisfecho con el modulo escogido para desarrollar las unidades didácticas y el 5,08 \% satisfecho .como puede apreciarse las respuestas se encuentran el rango de muy satisfecho y satisfecho.

A partir de los resultados obtenidos, se concluye que es factible de aplicación el Microdiseño de las unidades didácticas. 


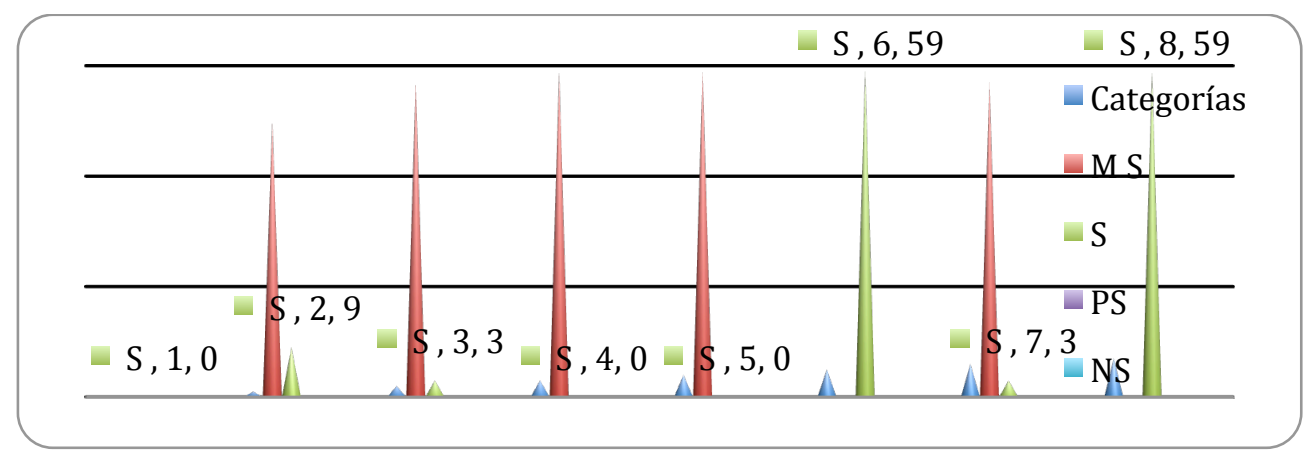

Figura 1. Resultados de la encuesta de satisfacción

\section{DISCUSIÓN}

La utilización del Microdiseño de las unidades didácticas en la formación del especialista de EGI, permite un mejor desarrollo del PEA, beneficia el crecimiento personal y profesional del residente para elevar la calidad del servicio de salud que se presta desde la Educación en el Trabajo, unido a la calidad de vida de la población.

La correcta planificación y selección de los contenidos, el cumplimiento de los objetivos propuestos, mediante la utilización de métodos y formas de organización que optimicen tiempo y recursos, con la participación activa y consciente de sus estudiantes en el Microdiseño de las unidades didácticas permite concretar el contenido interdisciplinario de la Estomatología dentro de las Ciencias de la Educación Médica.

La utilización de las TIC en la formación profesional, permite que el residente perciba mejor la realidad y no se quede en lo abstracto de lo que dijo el profesor como verdad absoluta.

En adición a lo anterior se observa una mayor incrementa el desarrollo de la creatividad y capacidad resolutiva, mejor organizacion del trabajo según los procesos lógicos del pensamiento. Pueden citarse: procesos de análisis, la síntesis, la abstracción y la generalización. Así como la comparación y clasificación de situaciones, hechos y problemas; aplicando formas de pensar inductivas y deductivas al igual que fomenta valores éticos-morales y de esta forma contribuir en la solución de los problemas que presenta el individuo, la familia o la comunidad.

En esta misma línea el Microdiseño de las unidades didácticas planteadas los contenidos son tanto teóricos como prácticos, están interrelacionados y responden a situaciones motivantes (se hace referencia a la motivación intrínseca), problematizantes, contextualizadas y significativas para los residentes ya que son cercanas a sus intereses, a su vida cotidiana, profesional y a sus experiencias.

Relacionado con la factibilidad de aplicación de las unidades a partir de los resultados obtenidos, cabe destacar que esta se consideró factible de aplicación, lo cual se encuentra en correspondencia con una de las características de la educación médica de posgrado ${ }^{(10,11)}$, que tiene como fin profundizar y ampliar los conocimientos para el perfeccionamiento teórico, metodológico y práctico de la labor profesional.

Desde esta perspectiva, la aplicabilidad del resultado es importante, si se tiene en cuenta que se estructuraron las unidades didácticas con profundidad, con la intención que la formación del especialista de EGI responda a su encargo social ${ }^{(12)}$.

Como complemento, se constata la factibilidad de aplicación de las unidades didácticas en la formación de especialistas, pues permite a todos los implicados interrelacionar los contenidos con la reflexión sistemática sobre las vivencias, experiencias desde los referentes teóricos asumidos y contextualizados ${ }^{(13)}$. 
Cabe resaltar que los resultados alcanzados por Quintero, Gort y Verona (14) en su trabajo corroboró el impacto positivo causado por la alternativa metodológica utilizada, demostrándose así la importancia y utilidad de la misma.

Es incuestionable referenciar los resultados obtenidos por Travieso ${ }^{(8)}$ con relación a la factibilidad de su propuesta a través de la pertinencia y coherencia de la misma.

Finalmente se puede concluir que la alternativa del Microdiseño de unidades didácticas aplicadas a la formación del especialista de EGI, articula lo científico, lo atencional y lo docente con el propósito de producir aprendizajes que se traduzcan en formas transformadoras de pensar, sentir, valorar y actuar, frente a los problemas de salud bucal que plantea la formación de recursos humanos en Estomatología, y la Educación Médica ${ }^{(15,16)}$.

Contribución de autoría: ACLV, MEAL Y LTR diseñaron el estudio, participaron en la recolección y análisis de datos; redacción del artículo. Todos los autores aprobaron el manuscrito.

\section{Fuente de financiamiento: Autofinanciado}

Conflicto de interés: Los autores declaran no tener conflicto de interés en la publicación de este artículo.

\section{REFERENCIAS}

1. Álvarez, C. M. y Fuentes, H. El postgrado. Cuarto nivel de Educación. Santiago de Cuba: Universidad de Oriente. Centro de Estudios de Educación Superior, 1996.

2. Seguí Ulloa A, Barciela González Longoria MC, Valdivié Provenza JR, Grau León IB. Perfeccionamiento del plan de estudio de la especialidad de Estomatología General Integral. Educ Med Super. 2014; $28(2$ ): 282291.

3. Cuba. Ministerio de Educación Superior. Reglamento de la Educación de Posgrado de la República de Cuba. Resolución Ministerial No. 132/2004. Gaceta Oficial de la República de Cuba, edición extraordinaria. 2004:51-8.

4. Salas Perea RS, Díaz Hernández L, Pérez Hoz G. El currículo de formación de especialistas médicos basado en competencias laborales. Educ Med Super. [Internet] 2013 jul-sep. [Citado 25 ene 2018]; 27(3): [aprox. 7p.]. Disponible en: http://scielo.sld.cu/scielo.php?script=sciarttext \&pid=s0864-21412013000300012.
5. Díaz Pérez CA. Modelo didáctico del proceso de formación de competencias profesionales específicas en la especialidad de Estomatología General Integral. [Tesis] Guantánamo: Universidad de Ciencias Médicas de Guantánamo; 2013 [citado 12 de septiembre 2018]. Disponible en: http://tesis.repo.sld.cu/697/1/TESIS_COMPE TENCIAS.pdf.

6. Fernández Vidal Ana, Díaz Quiñones José, Enrique Vilaplana Silvia. The Graduate Pedagogical Process in the Comprehensive General Medicine Specialization: towards a Practice based on the Cultural-historical Approach to Human Development. Medisur [Internet]. 2016 Ago [citado 2019 Sep 02] ; 14( 4 ): 447-455. Disponible en: http://scielo.sld.cu/scielo.php?script=sci_artte xt\&pid=S1727-897X2016000400012\&Ing=es.

7. Marchán-Carvajal Iván, Sanmartí Neus. Criterios para el diseño de unidades didácticas contextualizadas: aplicación al aprendizaje de un modelo teórico para la estructura atómica. Educ. quím [revista en la Internet]. 2015 [citado 2019 Sep 20], 26( 4 ): 267-274. Disponible en: http://www.scielo.org.mx/scielo.php?script=sc i_arttext\&pid=S0187893X2015000400267\&lng=es.

8. Travieso Ramos Nadina. Investigación educativa para la formación de posgrado de docentes en la educación médica. MEDISAN [Internet]. 2018 Mayo [citado 2019 Sep 02]; 22(5):498-507. Disponible en: http://scielo.sld.cu/scielo.php?script=sci_artte xt\&pid=S1029-30192018000500007\&lng=es.

9. Camaño Aureli .Hacer unidades didácticas: una tarea fundamental en la planificación de las clases de ciencias [revista en la Internet]. Abr 2013 [citado 2019 Sep 20] ; (74):7 Disponible

en:https://www.researchgate.net/profile/Aureli Caamano/publication/280382898 Hacer un idades_didacticas_una_tarea_fundamental_e n_la_planificacion_de_las_clases_de_ciencia s/links/55b368ae08aed62 1 ddfeeb63/Hacerunidades-didacticas-una-tarea-fundamentalen-la-planificacion-de-las-clases-deciencias.pdf.

10. Vargas Segura R. Acompañamiento formativo: una estrategia para la formación en investigación educativa de directivos, docentes y asesores de educación básica y superior. Rev Entreideias, Salvador. 2015; 4(1): 35-49.

11. Sosa Palacios $O$, Valdespino Alberti $A$, Martínez Ortega R, Broche Candó R, Fortún Sosa R, Núñez Alemán $G$, et al. Libro electrónico para la gestión del conocimiento de la investigación científica en el posgrado de especialidad en Ciencias Médicas. MEDISUR. 2014 [citado 27 agos 2019]; 12(6):904-16 
12. Colectivo de autores. Didáctica del posgrado Pedagogía. ICCP. La Habana: Editorial Pueblo y Educación; 2014.

13. Díaz, V. Didáctica y prácticas en posgrado: una aproximación teórica. UNIMAR. 2016. [citado 7 sep 2019]; 34(1):71-85.

14. Quintero Martí Coralia, Gort Hernández Magaly, Verona Ferro Teresa, Linares Miranda Marelis, Cordero Pérez José Luis. Alternativa metodológica para fortalecer el proceso enseñanza aprendizaje de Morfofisiología I en la carrera Enfermería. Rev Ciencias Médicas [Internet]. 2013 Dic [citado 2019 Sep 18] ; 17( 6 ): 164-179. Disponible en: http://scielo.sld.cu/scielo.php?script=sci_artt ext\&pid=S1561 31942013000600016\&lng=es.
15. Gamboa Graus, M., \& Cortina Bouver, V. Modelo para el diseño de unidades didácticas contextualizadas. Opuntia Brava, [Internet]. 2018. [citado 2019 Sep 20] 4(4): $\quad 55-68 . \quad$ Disponible en: https://doi.org/https://doi.org/10.35195/ob.v4 i4.437.

16. Franco Mariscal, Antonio; Ángel Blanco López; Enrique España Ramos. "El desarrollo de la competencia científica en una unidad didáctica sobre la salud bucodental. Diseño y análisis de tareas." Enseñanza de las ciencias: revista de investigación y experiencias didácticas [online], 2014, [citado 2019 Sep 18] 32(3) : 649-667. Disponible en https://www.raco.cat/index.php/Ensenanza/ article/view/287562/375715.

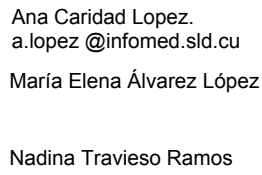

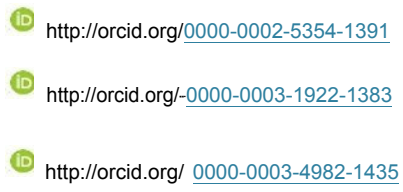

